\title{
Automated modeling of reflectance properties for industrial plant primitives
}

\author{
Brandon Baker and Cynthia Furse
}

\begin{abstract}
This paper outlines a method for determining reflectance parameters for modeling and rendering industrial plant primitives. The reflectance parameters are diffuse and specular material properties and the industrial plant primitives are pipes from raw LiDAR brightness data. For diffuse reflectance, the Lambertian model is compared to the Oren-Nayar model, and methods for estimating both are presented. For specular reflectance, the Phong model, commonly used in computer graphics, is used. The brightness data acquired from LiDAR systems are modeled as two different ideal cylinders to recover the diffuse and specular reflectance parameters and to illustrate the proposed method. A measured cylinder using brightness data acquired from a LiDAR scanner is also analyzed, and the diffuse and specular reflectance parameters are estimated. The estimate for the specular coefficient and exponent of the ideal cylinder are within $3.5 \%$ and $3.6 \%$, respectively and the estimate for the Lambertian reflectance albedo for the ideal cylinder is within $0.02 \%$ of the actual value. The estimate for the diffuse reflectance and roughness parameters for the second ideal cylinder are within $0.3 \%$ and $1.2 \%$, respectively. The estimated reflectance model of the measured cylinder have a mean relative error of $2.88 \%$ and a standard deviation of relative error of $4.0 \%$.
\end{abstract}

\section{Introduction}

As-built models of industrial plants are utilized extensively in asset management, asset virtualization, risk assessment, and emergency evacuation planning and training $[3,2,1]$. "As-builts", as they are commonly called, are databases containing existing condition information about a natural or human architected structure. Ascertaining as-built information for industrial plants usually involves taking measurements, utilizing those measurements to create geometric primitives (such as pipes, flanges, elbows etc.), annotating those primitives with descriptive information, and storing the results in a database for future use. This paper proposes methods for estimating 
specular and diffuse reflectance properties for pipes, the simplest and most prevalent of all industrial plant primitives.

The research conducted for this paper utilized Light Detection and Ranging (LiDAR) scanners to extract physical geometry and reflectance information from industrial plant pipes. LiDAR scanners are remote sensing devices that use laser pulses to detect the distance to an object [45], [46]. In addition to capturing 3D points, LiDAR scanners may also utilize amplitudes of the reflected laser pulses, and, if coupled with color sensors, color photographic data of physical objects. Such data contain vast amounts of information that is scarcely utilized to its full potential. For example, the visual aspect of the as-built information (if even available in a useful format) is underutilized in most circumstances [47]. Although many applications today can render massive amounts of 3D points (called "point clouds"), these systems do not exceed the visual quality of a polygonal 3D model, especially given the extreme capabilities of modern graphics hardware. Hence, point clouds are typically used to display and work with raw data, but not the final model.

Specular reflectance is the modeling of light as it reflects off a surface at a single (or very limited number of) angle(s). Specular reflectance describes an object's "shininess". Diffuse reflectance is the reflectance of light that scatters in many angles, is not "shiny," and is largely responsible for illustrating the contrast between brightness and darkness of the colors we see in objects.

Estimating specular and diffuse parameters has been performed for geometric modeling applications, where these two physical aspects of reflectance are determined independent of each other [39]. The purpose of determining the reflectance properties in that paper was to help determine the shape of the object under inspection. The shapes of primitives in industrial plants can be created in a similar manner using photogrammetric methods [3], a hybrid of photogrammetry and LiDAR scanners [5], or solely from LiDAR range images [4].

Once the geometry has been determined, the photorealistic attributes of geometric primitives are seldom utilized in industry today [1]. In fact, few existing computer aided design (CAD) visualization and utilization tools are even capable of properly rendering the specular and diffuse material properties of 3D CAD models [1]. Modern CAD models for industrial plants contain much more than simple geometric primitive information. Such information may include: material type, outer diameter, inner diameter, material density, melting point, specific heat, purchase price, purchase date, or life expectancy. If the specular and diffuse reflectance values of the industrial 
plant primitives in their current state were known, the material type could be automatically estimated. The material properties such as density, melting point, specific heat, life expectancy or even the inner diameter could be automatically estimated (when utilized in conjunction with geometric information such as a cylinder's outer radius) once they are identified with a matching material type in a database library. All the methods found in the literature that determine reflectance properties of an object do so utilizing photogrammetric methods. The object's shape and the light source(s) illuminating it are typically unknown, and the shape and light source have to be estimated along with the reflectance properties. The methods found in the literature that create CAD models of industrial plants focus on acquiring geometry; the visual aspect is of secondary concern. Furthermore, when a visual element is added to the model, raw photographic imagery is typically draped over the geometry as a texture map; no material reflectance properties are estimated $[4,5,3]$.

The methods outlined in this paper automatically extract specular and diffuse material properties from raw LiDAR data of pipes found in industrial plants. The sizes and spatial orientations of the pipes are assumed to be known, and the location and direction of the active light source emanating from the LiDAR scanner illuminating the pipes are also known. The determination of specular and diffuse reflectance properties is the sole objective of this paper, thus differing from related work found in the literature.

In section 2, more details regarding related work found in the literature is presented. Section 3 outlines some details of industrial plant modeling of cylindrical objects such as pipes, railings, supports, etc., where the specific mathematical representations of specular and diffuse reflectance models are provided and described. Section 3.1 illustrates the difference between the Oren-Nayar and Lambertian diffuse reflectance models [16].

The numerical inversion process used in this paper is described in section 4. The experimental results are outlined in section V. Reflective properties from two simulated ideal cylinders and a measured cylinder were determined. The first ideal cylinder was created to illustrate Phong specular [15] and Lambertian diffuse reflectance [10]. The second ideal cylinder was created to illustrate the differences between the Lambertian model and the more general Oren-Nayar model for diffuse reflectance. The final piece in this section outlines experimental results estimating specular and diffuse reflectance values of a measured cylinder using data acquired from a LiDAR scanner of an industrial plant pipe. Section VI contains conclusions and lists potential future work. 


\section{Related research}

Properties of light as they interact with an object and are perceived by human eyes have been researched for centuries $[10,11,12,13]$. Lambert offered a basic mathematical model that describes this interaction, and the Lambertian model is the most prevalent algorithm for computing diffuse reflectance today. The Lambertian model calculates the brightness of a particular point on an object based on the object's inherent color, the reflected angle of the light source, and its relation to the angle of the viewing direction. The visual properties of light, such as Lambertian reflectance, were applied to computer systems to enhance realism in computer graphics in the 1960s. Cook, Torrance, Sparrow, Phong, and Blinn contributed a great deal of pioneering work in the field $[44,14,15,16]$. The efficient approximation of specular reflectance is one aspect of early advances in computer graphics that has withstood the test of time.

Oren and Nayar introduced a generalization of Lambert's solution to the diffuse reflectance model by accounting for rough surfaces that scatter light more prevalently than other relatively smooth surfaces [16]. This contribution, although not as computationally efficient as the Lambertian model, provides a much more accurate representation for rough surfaces.

Applications utilizing diffuse and specular reflectance models have yielded advances in the field of determining the geometric properties of an object $[17,18,19,20,42,22,23,43,24,25,26,27,28,29,30,31,32,33,34]$. "Shape from shading," as it is more commonly called, has seen dramatic advances over the years. Specular highlights, specifically, can provide detailed and accurate information regarding the detailed shape of a surface in addition to its reflective properties. Nayar, et al. uses reflectance to perform object recognition [25].

The general science of specular and diffuse reflectance has seen significant attention. Brelstaff, et al. have utilized Lambertian constraints to detect specular reflection parameters [21]. Nayar et al. contributed additional work outlining the identification and removal of specular highlights using color and polarization [35]. Wolff, Nayar and Oren then published work that applies their enhanced reflectance models for computer vision [31]. Lin and Lee provide several methods for enhancing the visual appearance of objects utilizing specular and diffuse properties [36, 37, 38]. Ragheb, et al. illustrated a method for separating Lambertian and specular reflectance parameters for Machine Vision [39]. More recently, Nayar outlined a method for overcoming challenges in shape from shading techniques using a diffuse filter to alleviate specular highlights and shadowed regions common in digital images [40]. 


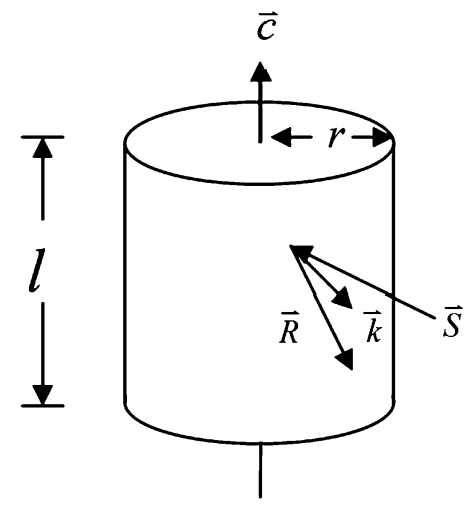

Figure 1: Geometric representation of cylinder model.

Other efforts have been made to model primitive objects found in industrial plants. Goulette automatically modeled CAD primitives found in industrial pipes using range images [4]. Hong similarly outlined how to obtain 3D models from industrial piping systems using digital photogrammetry in conjunction with laser scan data [5]. Chapman, et al. presented an omnidirectional imaging system for modeling industrial plants [3].

Previous methods for modeling industrial plant primitives focus on determining the objects' geometry - size of pipe, orientation in space, its clearance from other objects, etc. This paper uses LIDAR data similar to Goulette [4] and [5], but we determine diffuse and specular reflectance properties instead of primitive geometry. The reflectance properties determined by methods outlined in this paper may enable one to more render realistic models more efficiently and determine the nature and well being of primitive objects in industrial plants.

\section{Industrial plant cylinder modeling}

\subsection{Geometric representation}

One of the most common and most basic primitive forms in industrial plants is the cylinder. Pipes, railings, conduit, heating and cooling ducts, to name a few, are all cylindrical. The typical geometric aspects of a CAD model of a cylinder are shown in Figure 1 . We use a centerline vector, $\vec{c}$; a radius $r$; a starting point (not shown); and an extrusion or length, $l$; a surface normal vector, $\vec{k}$; a light source vector, $\vec{S}$; and a light reflection vector, $\vec{R}$. The reflectance properties for our model include: the specular coefficient, 


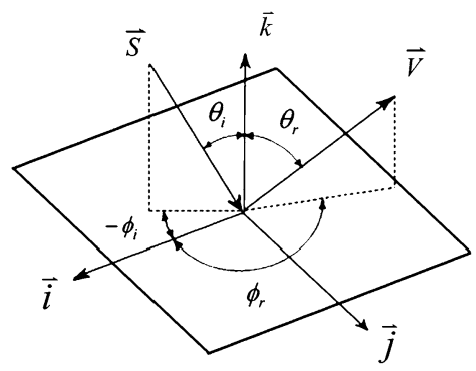

Figure 2: Geometric representation for Oren-Nayar model.

$S$ (a scalar), and exponent, $n$; and the diffuse coefficient (albedo), $\rho$, and surface roughness coefficient, $\sigma$.

Figure 2 shows some geometric elements used in the Oren-Nayar model. $\theta_{i}$ is the azimuth incident angle, $\theta_{r}$ is the azimuth reflected angle, $\phi_{i}$ is the polar incident angle, $\phi_{r}$ is the polar reflected angle, $\vec{k}$ and $\vec{S}$ are the same as in Figure 1, and $\vec{i}$ and $\vec{j}$ are orthogonal vectors tangential to the surface of the cylinder. A planar surface is shown in Figure 2, depicting a small area where the surface is a plane in the limit.

The specular brightness of a surface $\left(k_{\text {spec }}\right)$ is defined by the Phong model [15] given by

$$
k_{\text {spec }}=S(\vec{R} \cdot \vec{V})^{n}
$$

A sample (ideal) cylinder is created and used for sections 5.1 and 5.2. Various reflective properties are assigned to the ideal cylinder, to which noise is added, and then the assigned reflective properties are determined by numerical inversion. Next, in section 5.3, LiDAR data of an actual measured cylinder from an industrial plant are used to create a geometric model, from which the reflective properties are determined by numerical inversion.

Since LiDAR scanners typically operate in the infrared or near infrared spectrum, passive light in the visible spectrum (from overhead lights, for example) will not interfere with the near infrared active light source provided by the LiDAR scanner. Due to the nature of LiDAR scanners, the emitting and detecting origins are identical, and likewise, the emitting and detecting paths are coaxial.

The Lambertian diffuse reflectance ratio $L_{d}[1]$ can be expressed as:

$$
L_{d}=\frac{L_{r}}{L_{i}}=\frac{\rho}{\pi} \cos \left(\theta_{i}\right)
$$


where $L_{r}$ is the red, green or blue color value received at the sensor for a color imaging device, or the brightness value received in the LiDAR case (where the intensity of the reflected light is mapped in grayscale); $L_{i}$ is the intensity of light emanating from the source; $L_{d}$ is the ratio of reflected light versus emitted light; $\rho$ is the diffuse coefficient (albedo); $\theta_{i}$ is the azimuth incident angle; and $L_{r}$ is the resulting brightness. Typically, a LiDAR scanner will record the ratio $L_{d} / L_{i}$ as a unitless reflectance ratio, so we will use that quantity as the diffuse contribution to the "brightness" or the diffuse component of the "data vector." In this paper we utilize the quantity $\rho / \pi$ frequently for convenience, even though $\rho$ is the "albedo."

The Oren-Nayar generalized reflectance model [16] can be expressed as:

$$
L_{d}=\frac{\rho}{\pi} \cos \left(\theta_{i}\right)\left(A+\left(B \max \left[0, \cos \left(\phi_{i}-\phi_{r}\right)\right] \sin (\alpha) \tan (\beta)\right)\right.
$$

where

$$
\begin{gathered}
A=1-0.5 \frac{\sigma^{2}}{\sigma^{2}+0.33} \\
A=1-0.5 \frac{\sigma^{2}}{\sigma^{2}+0.33} \\
\alpha=\max \left[\theta_{r}, \theta_{i}\right], \text { and } \\
\beta=\min \left[\theta_{r}, \theta_{i}\right]
\end{gathered}
$$

where $\rho$ is the diffuse coefficient (albedo), and $\sigma$ is the surface roughness coefficient.

The Oren-Nayar reflectance model (3) simplifies to the Lambertian model (2) when $\sigma=0$. An additional simplification can be made since the emitting and detecting axes and origins are identical for LiDAR scanners. This suggests that $\theta_{i}=\theta_{r}$ and $\phi_{i}=\phi r$; thus, (3) reduces to

$$
L_{d}=\frac{\rho}{\pi} \cos \left(\theta_{i}\right)(A+(B \sin (\alpha) \tan (\beta)) .
$$

\subsection{Ideal cylinder with Oren-Nayar diffuse reflectance}

Brightness values for an ideal cylinder with Lambertian diffuse reflectance and an ideal cylinder with Oren-Nayar diffuse reflectance (both without specular reflectance) are shown in Figure 3 to emphasize the difference between the two models. The brightness values arranged as pixels in an image are shown in Figure 3. These brightness values are the data vector values, $d$, that will be used in the inversion process in the next section. 

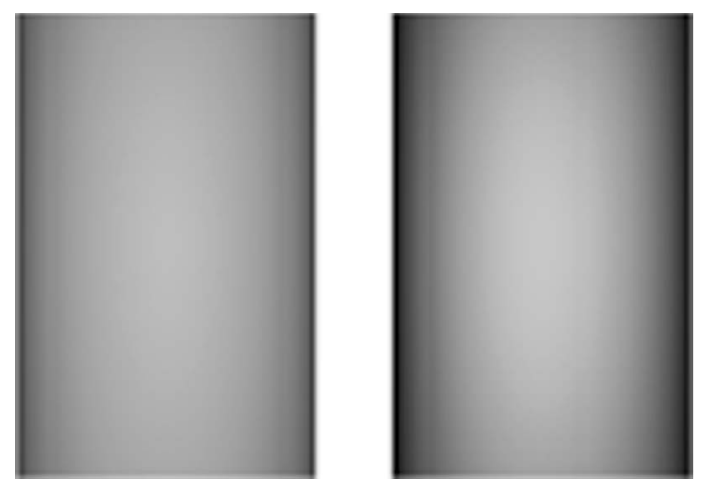

Figure 3: Brightness values, $d$, of ideal cylinders: Oren-Nayar diffuse model (left), $\rho / \pi=0.5, \sigma=0.25, S=n=0$; and Lambertian model (right); $\rho / \pi=0.5, \sigma=0.0, S=n=0$.

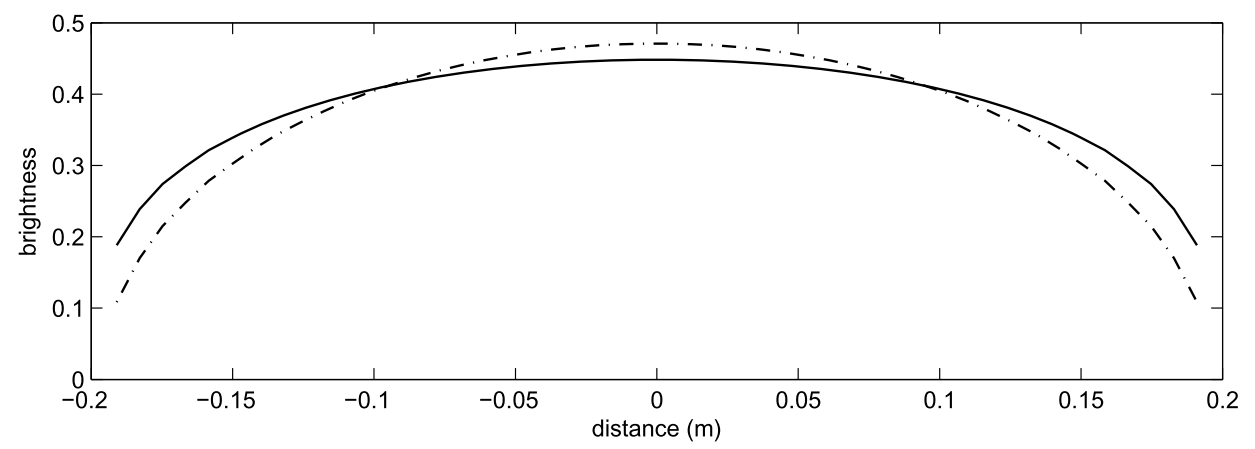

Figure 4: Statistical mean for Lambertian reflectance (---) and Oren-Nayar (-) along a cross section of the pipe.

Figure 4 shows brightness values along a cross section of the pipe, with the mean of intensity values at a given horizontal distrance from the pipe center plotted for the Oren-Nayar and the Lambertian models. The intensity rounds off gradually as the angle of incidence strays from the center for the Lambertian reflectance; whereas, the Oren-Nayar model (represented as a dash-dotted line) tapers off slowly around the center of the pipe, and then drops abruptly at the edges.

Figure 4 illustrates the reflectance values shown in Figure 3 that exist at different radial distances from the center. As shown in Figure 3, Figure 4 shows the gradual rounds off effect inherent in the Lambertian reflectance; whereas, the Oren-Nayar model demonstrates the slow tapering off around 
the center of the pipe, and then abruptly drops at the edges. Whereas the Oren-Nayar is more computationally intense, it also provides a more general characterization of an object's diffuse reflectance properties, so it should provide added information that the Lambertian does not.

\section{Numerical inversion process}

Numerical inversion is an iterative process by which a set of unknown model parameters, $m$, can be estimated by using an initial guess of the model parameters and a known forward operator, $A(m)$. The forward operator provides an approximation, $\hat{d}$, to the data vector, $d$, and the difference between the $d$ and $\hat{d}$ (in some sense) provides an update to the model parameters for the next iteration. The forward operator for the inverse problems found in this paper is

$$
\hat{d}=A(m)=k_{\text {spec }}(S, n)+L_{d}\left(\frac{\rho}{\pi}, \sigma\right),
$$

where $k_{\text {spec }}$ and $L_{d}$ are determined by (1) and (8) respectively, and the model parameter vector, $m$, is $S, n, \rho, \sigma$.

The forward operator for this problem is a non-linear process, so a nonlinear inversion technique must be employed. Conjugate gradient, steepest descent and Newton's method are a few possible inversion techniques that may be used to solve this problem. For this paper, Newton's method was selected as the numerical inversion algorithm based on its reputation for rapid convergence.

Ideal cylinder \#1 was modeled using Phong specular (1) and Lambertian diffuse reflectance (8). The model parameter vector $S, n, \rho, \sigma$ for ideal cylinder \#1 has specular content $(S=0.154$, and $n=26.8)$, and a Lambertian reflectance coefficient of $\rho / \phi=0.498$. For this cylinder, (9) becomes:

$$
A_{L}(m)=S(\vec{R} \cdot \vec{V})^{n}+\frac{\rho}{\pi} \cos \left(\theta_{i}\right)
$$

where $\sigma=0$. We created the ideal model using brightness data calculated by (10) with the parameter values given above, then added $1.45 \%$ standard deviation of Gaussian noise.

Ideal cylinder \#2 was modeled using only Oren-Nayar diffuse reflectance (8) without a specular component $\left(k_{\text {spec }}=0\right)$ to illustrate the differences between the Lambertian model and the Oren-Nayar model. For the numerical inversion of this cylinder (9) becomes:

$$
A_{O N}(m)=\frac{\rho}{\pi} \cos \left(\theta_{i}\right)(A+B \sin (\alpha) \tan (\beta)) .
$$


where $\mathrm{m}=[\rho, \sigma] ; A$ and $B$ are computed by (4) and (5), and $\alpha$ and $\beta$ are determined by (6) and (7).

Finally, the measured cylinder from LiDAR intensity data was modeled using specular and Oren-Nayar model parameters. Newton's method for inverse problems iteratively updates $m$ according to

$$
m_{i}=-i n v\left(F^{\prime} F\right) F^{\prime}\left(\hat{d}_{i-1}-d\right) \text {. }
$$

where $F$ is the Frechet derivative of the forward operator, $A$; and $d$ is the brightness data vector. More information on the Frechet derivative, uniqueness and Newton's method can be found in $[6,7,8]$.

The model parameters were initialized according to:

$$
\begin{gathered}
S_{o}=\max (d)-\text { median }(d) \\
n_{o}=20 \\
\rho_{o}=\text { median }(d) \\
\sigma_{o}=0.1
\end{gathered}
$$

The exit criterion for the inversion process is met when the change in the misfit between iterations is less than 0.0145 divided by the number of elements in the data vector; this corresponds to an average error of $1.45 \%$ per datum. LiDAR scanner manufacturers don't publish error metrics of brightness data, so we simply estimate this to be $1.45 \%$.

\section{Experimental results}

\subsection{Ideal Lambertian and specular model}

Figure 5 shows the data vector, $d$, from ideal cylinder \#1 and the estimate of the data vector, $\hat{d}$, from the inverted model.

The inversion process, in the presence of noise, was able to estimate the two quantities for specular reflectance (the specular coefficient $S$ and the exponent $n$ ) within $3.5 \%$ and $3.6 \%$ of their actual values, respectively, and the albedo to within $0.02 \%$, as shown in Table $1 .(\sigma=0$ in $(1)$, so the final column on the table was not estimated.)

These results correspond reasonably well to the $1.45 \%$ standard deviation of noise added to the data vector. The effect of the specular component only affects a relatively small portion of the surface area of the cylinder (where the bright highlights appear on Figure 5), and therefore, a larger specular reflectance error is understandable. Contrarily, the diffuse coefficient affects the entire visible surface of the cylinder; therefore the error is minimized in the presence of zero mean noise. 

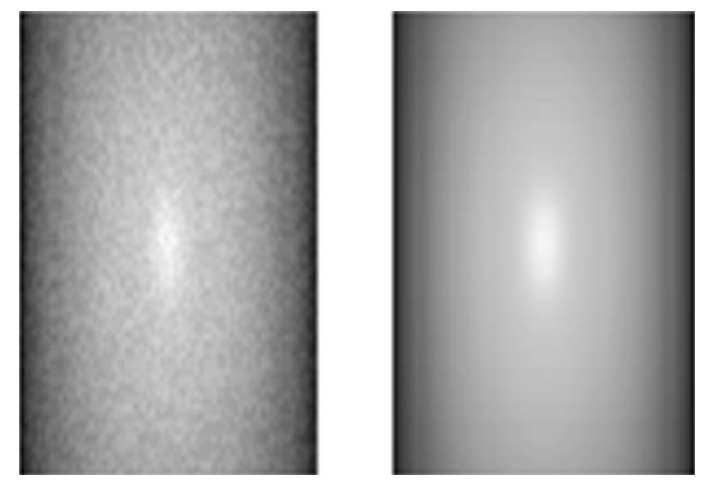

Figure 5: Ideal cylinder \#1: data vector, $d$, of Lambertian diffuse and Phong specular reflectance with noise (left), and inverted estimate, $\hat{d}$ (right).

Table 1: Inverted Model Vector, $m$, for Ideal Lambertian Cylinder \#1. Values for the specular coefficient $(\mathrm{S})$, specular exponent $(\mathrm{n})$, and diffuse reflectance $(\rho / \pi)$ for the Ideal and Estimated cylinders are shown. The absolute value of the relative error is also listed for each variable. Oren-Nayar roughness $(\sigma)$ is not used for Lambertian reflectance models

\begin{tabular}{ccccc}
\hline Type & $S$ & $n$ & $\rho / \pi$ & $\sigma$ \\
\hline Ideal & 0.150 & 27.0 & 0.500 & - \\
Estimate & 0.145 & 25.9 & 0.499 & - \\
$\mid$ rel error $\mid$ & $3.5 \%$ & $3.6 \%$ & $0.02 \%$ & - \\
\hline
\end{tabular}

\subsection{Ideal Oren-Nayar cylindrical model}

The second ideal model (ideal cylinder \#2) uses the Oren-Nayar cylindrical model (8) and provides a more generalized estimation of diffuse reflectance phenomena than the Lambertian model by accounting for rough surfaces that exhibit a broader dispersion of light due to surface roughness. Figure 6 shows the noisy model (left) compared to the inverted estimate (right).

The ideal noisy cylinder and the inverted approximation have a similar appearance. The diffuse albedo was estimated within $0.3 \%$ of the actual value and the roughness was within $1.2 \%$ as shown in Table 2.

The numerical inversion process described in this paper for estimating reflectance parameters for cylinders (industrial pipes) has proven to effectively determine reflectance parameters in the presence of noise for ideal cylinders. In this section we considered ideal cylinder models with noise added to the data. In the next section we perform a similar operation using measured data on a metal pipe found in an industrial plant. 

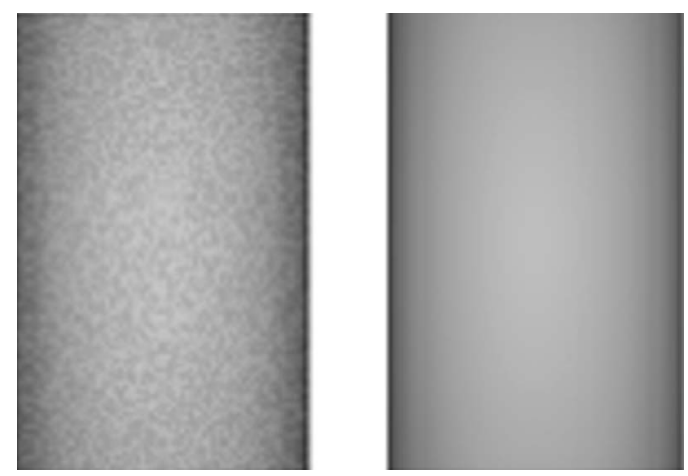

Figure 6: Ideal cylinder \#2: data vector, $d$, of Oren-Nayar diffuse reflectance with noise (left), and inverted estimate, $\hat{d}$ (right).

Table 2: Inverted Model Vector, $m$, for Ideal Cylinder \#2. Values for the diffuse reflectance $(\rho / \pi)$ and Oren-Nayar roughness $(\sigma)$ for the Ideal and Estimated cylinders are shown. The absolute value of the relative error is also listed for both variables. Values for the specular coefficient $(\mathrm{S})$, specular exponent (n) were not used for these Oren-Nayar models

\begin{tabular}{ccccc}
\hline Type & $S$ & $n$ & $\rho / \pi$ & $\sigma$ \\
\hline Ideal & - & - & 0.500 & 0.250 \\
Estimate & - & - & 0.498 & 0.243 \\
|rel error & - & - & $0.3 \%$ & $1.2 \%$ \\
\hline
\end{tabular}

\subsection{Measured cylinder}

In order to evaluate the Oren-Nayar and Lambertian models in an actual industrial application, LiDAR measurements of a metal pipe vertically aligned, had a radius of $0.20 \mathrm{~m}$, and was covered in light blue paint that exhibited a fair amount of specular reflectance. The LiDAR scanner used was a Leica C10 [9] The diffuse roughness $(\sigma)$ of the measured pipe was assumed to be non-zero, so the more general Oren-Nayar diffuse model 11 was used for the inversion process. The albedo $(\rho)$ was 0.498 ; the measured roughness $(\sigma)$ was 0.237 . The measured specular coefficient $(S)$ was 0.154 , and the measured specular exponent $(n)$ was 26.8 .

A cross sectional scatter-plot with all of the brightness values examined for the measured pipe is shown in Figure 7 . The statistical mean $d_{m}$ of the data vector, $d$, and the statistical mean $m$ of the inverted estimate of the data vector, $\hat{d}$, along the same cross section are shown in Figure 8 . The error $\left(\left|d_{m}-\hat{d}_{m}\right|\right)$ for the entire cross section of the pipe is shown in Figure 9, and 


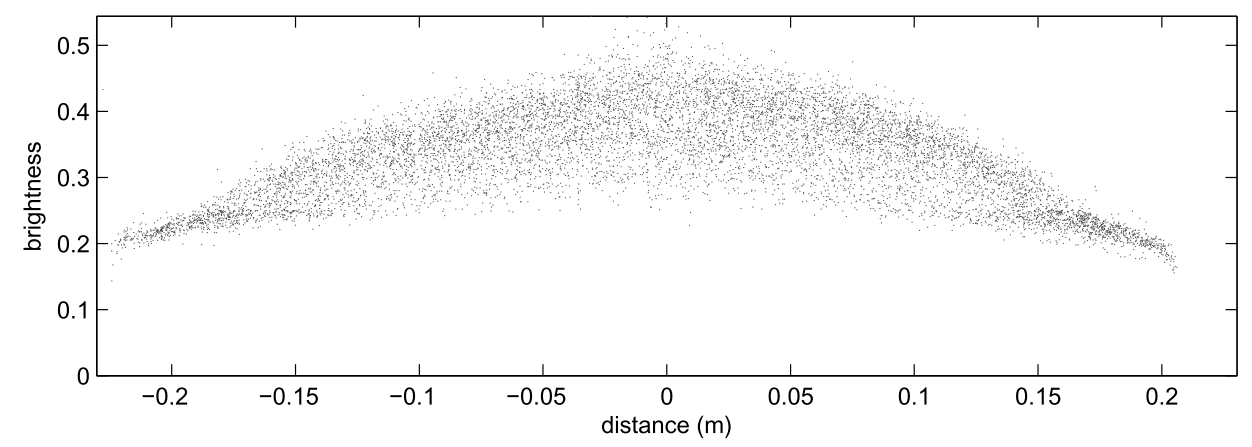

Figure 7: Cross section of brightness data vector, $d$, of measured cylinder.

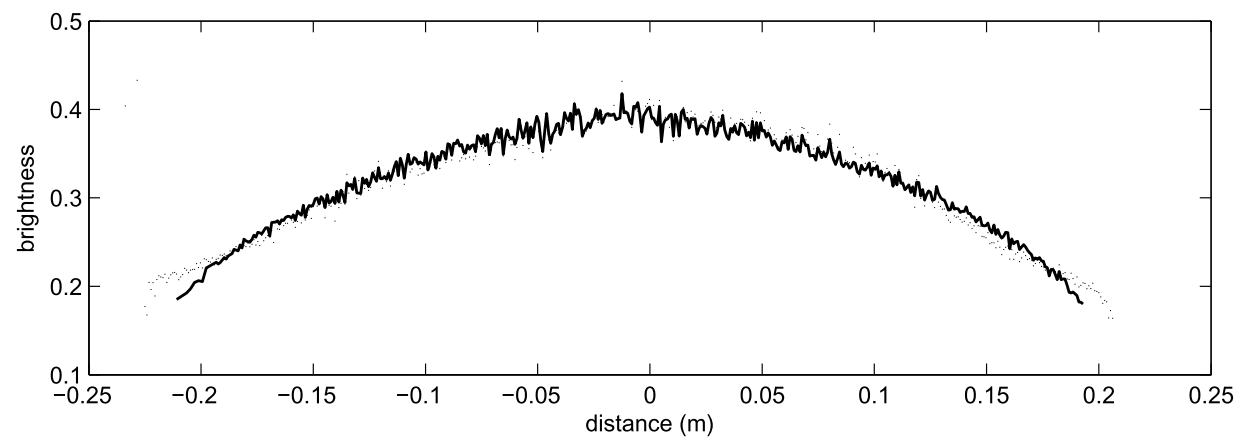

Figure 8: Cross section of statistical mean of data vector, $d_{m}$ (dots) and numerical inversion estimate $\hat{d}_{m}$ (line).

the relative error for the entire cross section of the pipe $\left(\left|d_{m}-\hat{d}_{m}\right| / \hat{d}_{m}\right)$ is shown in Figure 10.

The inverted values closely resemble the brightness data vector near the center of the pipe where the specular reflection and diffuse reflectance values are maximized. The estimate deviates significantly near the edge of the pipe where the brightness drops below $20 \%-25 \%$ reflectance. This is most likely caused by the nonlinear dynamics of the receiver circuitry. LiDAR scanners possess input signal amplifiers to compensate for the limited dynamic range in the analog to digital conversion process. When the input signal drops below a specified threshold, the analog amplifier boosts the input signal so a reading can be made. The estimate of the roughness coefficient was most adversely affected by this added circuitry. The inversion process attempts to estimate the brightness of the pipe at the edges, but this nonlinear phenomenon is not accounted for in the estimator model 9. 


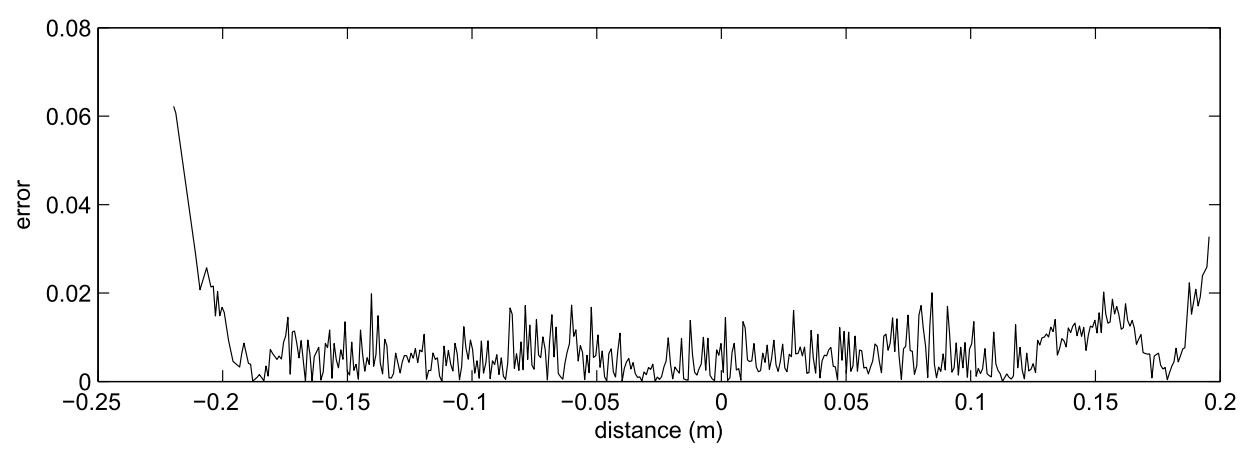

Figure 9: Cross section of absolute error of statistical mean $\left|d_{m}-\hat{d}_{m}\right|$.

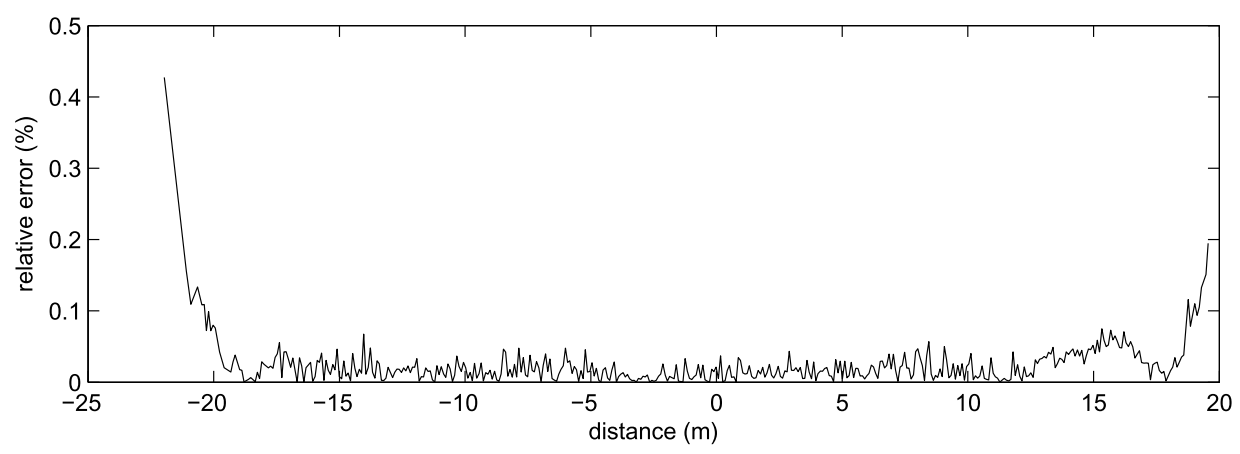

Figure 10: Cross section of relative error $\left|d_{m}-\hat{d}_{m}\right| / \hat{d}_{m}$.

The inverted value for the specular highlight closely matches the brightness data vector. The equation for the specular reflectance 1 is used extensively in computer graphics and is not derived from any physics equation, but Figure 8 shows that it is a close approximation to the actual physical phenomenon exhibited by light reflecting off of specular reflective surfaces.

The mean relative error (mean of brightness values in Figure 10) is $2.88 \%$ and the standard deviation of the relative error is $4.0 \%$. The values for each of the inverted model parameters are shown in Table 3.

A photograph of the measured cylinder, its measured LiDAR reflectance values, and the approximated reflectance values obtained by numerical inversion are shown in Figure 11, arranged as pixels in an image array.

The photograph demonstrates visual anomalies inherently found in circumstances involving passive light sources. Although there are many light sources present, the dominant light source is above and to the right of the 
Table 3: Inverted Model Vector, $m$, for Measured Cylinder. Values for specular coefficient (S), specular exponent (n), diffuse reflectance $(\rho / \pi)$, and Oren Nayar roughness $(\sigma)$ are given. Actual values are not known for this physical pipe

\begin{tabular}{ccccc}
\hline Type & $S$ & $n$ & $\rho / \pi$ & $\sigma$ \\
\hline Estimated & 0.154 & 26.835 & 0.498 & 0.237 \\
\hline
\end{tabular}

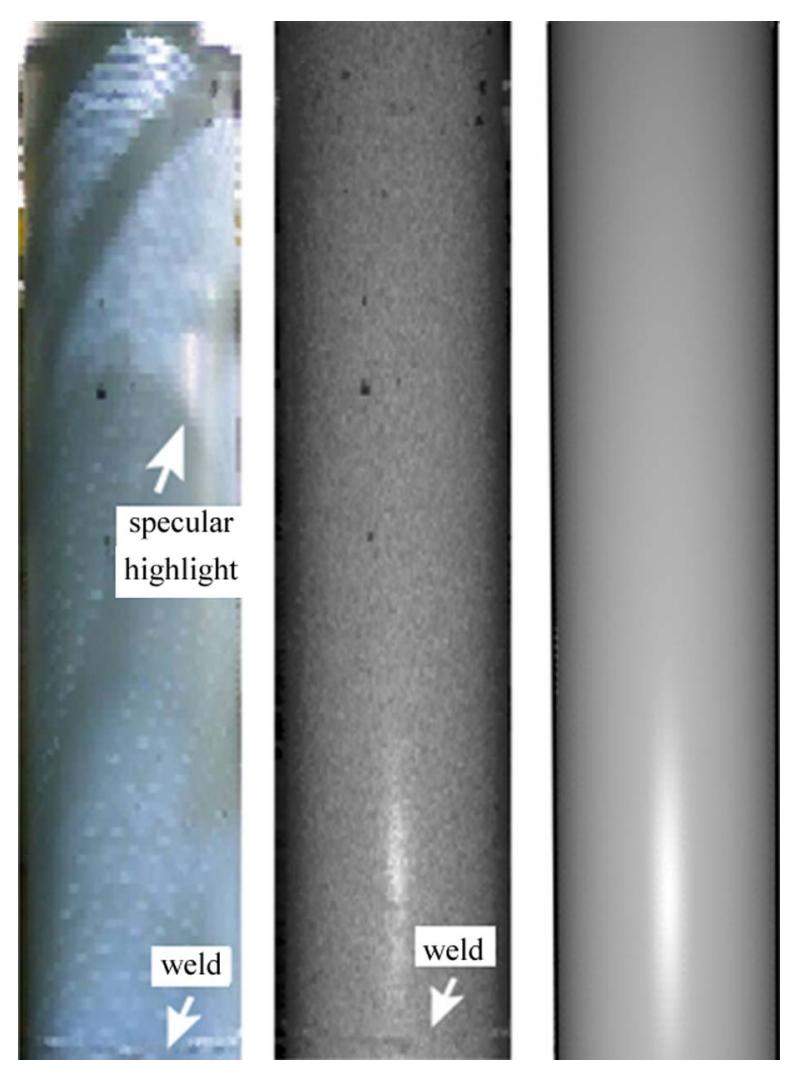

Figure 11: Photograph of measured pipe (left); LiDAR brightness data vector, $d$, (center); and inverted brightness estimate, $\hat{d}$ (right).

pipe, and there are shadows (and highlights) of other pipes and metal grates visible. A specular highlight appears on the right-hand side of the pipe, just above the middle, as labeled on Figure 11. Most of the other variations in brightness are due to highlights and shadows demonstrated by diffuse reflectance.

The center image is LiDAR brightness of the measured pipe. There are obvious anomalies in the actual brightness data due to noise in the receiver 
circuitry of the LiDAR scanner. One may observe that there are obvious dark regions of similarities between the visible light image (left) and the LiDAR brightness (center). Both sensors failed to return adequate brightness in those regions due to a foreign substance on the pipe in those regions. Also, there is a weld near the bottom of the pipe that is visible in both measured images that is not present in the estimated image (right).

\section{Conclusions}

A method for determining the specular and diffuse reflectance properties for two ideal and one measured pipe from an industrial plant was presented. LiDAR data intensity values provided the brightness data for the measured cylinder. The reflectance properties determined include the specular coefficient and specular exponent commonly used in computer graphics, as well as the diffuse albedo parameter and the Oren-Nayar roughness parameter.

Newton's method from numerical inversion was used to compute the estimate for the model parameters. The inversion process for the ideal cylinders recovered the model parameters within $3.6 \%$ of the actual values, providing a suitable method for recovering reflectance parameters.

Inversion of the model parameters for the measured cylinder provided an accurate estimate to the actual data to within $3.5 \%$ for all brightness values over 0.3 . The relative errors associated with brightness estimation for data at the extreme edges of the pipe, where the incident angle is close to orthogonal to the surfaced normal, were as high as $44.7 \%$ due to nonlinear amplifiers in the detection hardware.

\section{Acknowledgements}

We would like to thank McNeil Engineering and Calabi Yau Systems [41] for providing the industrial plant data.

\section{References}

[1] C. Toro and J. Posada (2006). Improving virtual reality applications in CAD through semantics. The Intl. Journal of Virtual Reality, 5, no. 4, 39-46.

[2] C. Jorge (2010). Nuclear plants and emergency virtual simulations based on a low-cost enginer reuse. Nuclear Power [Online], available: http://www.intechopen.com/books/nuclear-power/nuclear-plants-andemergency-virtual-simulations-based-on-a-low-cost-engine-reuse. 
[3] D. Chapman (2004). An omnidirectional imaging system for the reverse engineering of industrial facilities. The Intl. Archives of the Photogrammetry, Remote Sensing and Spaitial Information Sciences, 34, no. XXX.

[4] F. Goulette (1997). Automatic CAD modeling of industrial pipes from range images. Proc. Intl. Conf. on Recent Advances in 3-D Digital Imaging and Modeling, Ottawa, Ontario, Canada.

[5] J. Hong (2003). 3D modelling of industrial piping systems using digital photogrammetry and laser scanning. M.S. Thesis, Dept. Elec. Eng., Ryerson, Univ. Ottawa, Ontario, Canada.

[6] J. Oliensis (1991). Uniqueness in shape from shading. Intl. Journal of Computer Vision, 2, no. 2, 75-104.

[7] M. Zhdanov (2002). Geophysical Inverse Theory and Regularization Problems. Elsevier Science, New York, New York.

[8] A. Tarantola (2005). Inverse Problem Theory and Methods for Model Parameter Estimation. Philadelphia, PA, SIAM. MR2130010

[9] Leica Geosystems (2012). [Online], http://hds.leica-geosystems.com/ downloads123/hds/hds/ScanStation\%20C10/brochures-datasheet/ Leica_ScanStation_C10_DS_en.pdf. Accessed August 26, 2015.

[10] J. Lambert (1760). Photometria sive de mensure de gratibus liuminis, colorum umbrae. W. Engelmann, Leipzig, Germany.

[11] M. Kerker (1969). The Scattering of Light and Other Electromagnetic Radiation. New York, Academic.

[12] L. I. Mandelstam (1926). Light scattering by inhomogeneous media. $Z$ h. Russ. FizKhim Ova, 381.

[13] P. Kubelka (1931). Ein Beitrag zur Optik der Farbanstriche. Zeits. f. Techn. Physik, 12, 593-601.

[14] R. Cook (1982). A reflectance model for computer graphics. ACM Transactions on Graphics, 1, no. 1, 7-24.

[15] B. Phong (1975). Illumination for computer generated pictures. Communications of the ACM, 18, no. 6, 311-317.

[16] M. Oren and S. Nayar (1995). Generalization of the Lambertian model and implications for machine vision. International Journal of Computer Vision, 14, no. 3, 227-251. 
[17] B. K. P. Horn (1978). Determining shape and reflectance using multiple images. Massachussetts Institute of Technology, 113-115.

[18] J. J. Koenderink (1979). The internal representation of solid shape with respect to vision. Biological Cybernetics, 32, 211-216.

[19] B. K. P. Horn (1986). The variational approach to shape from shading. Computer Vision, Graphics, and Image Processing, 33, no. 2, 174208.

[20] G. H. Healey (1987). Local shape from specularity. IEEE Intl. Conf. on Computer Vision, 151-160.

[21] G. Brelstaff (1988). Detecting specular reflectoin using Lambertian constraints. IEEE Intl. Conf. on Computer Vision, 297-302.

[22] M. J. Brooks (1989). Shape from Shading, BKP Horn, MIT Press, Cambridge, MA. MR1062877

[23] S. K. Nayar (1990). Shape from interreflections. Intl. Jornal of Computer Vision, 6, no. 3, 173-195.

[24] J. J. Koenderink (1992). Surface shape and curvature scales. Image and Vision Computing, 10, no. 8, 557-565.

[25] S. K. Nayar (1996). Reflectance based object recognition. Intl. Journal of Computer Vision, 17, no. 3, 219-240.

[26] J. Lu (1999). Reflectance and shape from images using a collinear light source. Intl. Journal of Comptuer Vision, 32, no. 3, 213-240.

[27] Q. Zheng (1991). Estimation of illumination direction, albedo, and shape from shading. Computer Vision and Pattern Recognition, 540545.

[28] S. Savarese (2001). Local analysis for 3D reconstruction of specular surfaces. Computer Vision and Pattern Recognition, 2, 738-745.

[29] H. Ragheb (2002). Diffuse reflectance models for rough surfaces: a geometrical study for shape-from-shading. Proc. The 2nd Intl. Workshop on Texture Analysis and Synthesis, Copenhagen, Denmark, 113-116.

[30] B. Mercier (2007). A framework for automatically recovering object shape, reflectance, and light sources from calibrated images. Intl. Journal of Computer Vision, 73, no. 1, 77-93.

[31] L. Wolff (1998). Improved diffuse reflection models for computer vision. Intl. Journal of Computer Vision, 30, no. 1, 55-71. 
[32] T. Yu (2007). Shape and view independent reflectance map from multiple views. Intl. Jorunal of Comptuer Vision, 73, no. 2, 123-138.

[33] K. Yoon (2010). Joint estimation of shape and reflectance using multiple images with known illumination conditions. Intl. Journal of Computer Vision, 86, no. 2-3, 192-210. MR2718753

[34] W. Smith (2010). Estimating facial reflectance properties using shapefrom-shading. Intl. Journal of Computer Vision, 86, no. 2-3, 152-170. MR2718751

[35] S. K. Nayar (1993). Removal of specularities using color and polarization. Computer Vision and Pattern Recognition, 583-590.

[36] S. Lin (1999). A representation of specular appearance. IEEE Intl. Conf. on Computer Vision, Kerkyra, Corfu, Greece, 849-854.

[37] S. Lin (1999). Estimation of diffuse and specular appearance. IEEE Intl. Conf. on Computer Vision, Kerkyra, Corfu, Greece, 855-860.

[38] S. Lin (2000). An appearance representation for multiple reflection components. Computer Vision and Pattern Recognition, 1, 105-110.

[39] H. Ragheb (2001). Separating labertian and specular reflectance components using iterated conditional modes. Proc. British Machine Vision Conf., Manchester, UK, 10-13.

[40] S. K. Nayar (2012). Diffuse structured light. IEEE Conf. on Computational Photography, Cluj-Napoca, Romania, 1-11.

[41] Calabi Yau Systems (2013). LD3 Studio [Online], http://www. calabiyausystems.com/Products-LD3Studio.aspx\#!page_products_ ld3studio.

[42] A. Blake and G. Brelstaff (1988). Geometry from specularities. IEEE Intl. Conf. on Computer Vision, 394-403.

[43] A. Blake and H. Bulthoff (1991). Shape from specularities: computation and psychophysics. Phil Trans R. Soc. Lond. B 331, 237-252.

[44] J. Blinn (1977). Models of light reflection for computer synthesized pictures. Computer Graphics, SIGGRAPH 1977, 187.

[45] S. Sangam (2012). Light detection and ranging [Online], http:// www.123seminarsonly.com/Seminar-Reports/022/52496573-LIDARIN-IEEE-FORMAT.docx (6.18.2015). 
[46] A. Wehr (1999). Airborne laser scanning - an introduction and overview. ISPRS Journal of Photogrammetry and Remote Sensing, 54, no. 2-3, 68-82.

[47] C. Toro (1996). Improving virtual reality applications in CAD through semantics. The International Journal of Virtual Reality, 5, no. 4, pp. $39-46$.

BRANDON BAKER

2176 E $3970 \mathrm{~S}$

SLC, UT 84124

$\mathrm{USA}$

E-mail address: brandon.baker@pinpoint3d.com

Cynthia Furse

John R PARK Building RoOm 210

201 PRESIDENT's CiRCle

UNIVERSITY OF UTAH

SLC, UT 84112

USA

E-mail address: cynthia.furse@utah.edu

Received 3 August 2013 